\title{
Role of Intraductal RFA: A Novel Tool in the Palliative Care of Perihilar Cholangiocarcinoma
}

\author{
Tobias J. Weismüller \\ Department of Medicine 1, University Hospital Bonn, Bonn, Germany
}

\author{
Keywords \\ Cholangiocarcinoma - Endoscopic retrograde \\ cholangiopancreatography · Endoscopy · Palliation . \\ Radiofrequency ablation
}

\begin{abstract}
Background: Patients with irresectable perihilar cholangiocarcinoma (PHC) have a limited prognosis with median survival times still less than 1 year. In addition to the current standard first-line systemic chemotherapy (gemcitabine and a platinum derivate), endoscopic treatment aims to ensure adequate drainage of the biliary system by placing biliary plastic or metal stents. Local ablative procedures like intraluminal biliary brachytherapy (ILBT) or photodynamic therapy (PDT) are used to improve local tumor control and to optimize the stent patency. Summary: Intraductal radiofrequency ablation (RFA) is another promising tool in the therapeutic armamentarium for the endoscopic management and tumor ablation of extrahepatic cholangiocarcinoma (eCCA). By applying thermal energy to the tissue through high-frequency alternating current, RFA induces coagulative necrosis and causes local destruction of the tumor. It is established as a first line percutaneous treatment of solid liver tumors, and since 2011 an endoscopic catheter is available that allows intraductal RFA in the biliary or pancreatic ducts. While the first pilot studies primarily evaluated this new method in patients with distal eCCA, there is now evidence accumulating also for PHC. Two retrospective and two prospective studies demonstrated a significantly improved overall survival and a longer stent patency with intraductal RFA, which overall had a favorable safety profile and was not associated
\end{abstract}

with a significant increase in adverse events. However, prospective studies comparing the efficacy and safety of intraductal RFA, PDT, and/or ILBT are lacking. Key Messages: Recent studies suggest that intraductal RFA is an effective and well-tolerated additional treatment option with regard to stent patency but also overall survival. Since RFA has fewer systemic side effects and requires less logistical effort when compared to ILBT and PDT, intraductal RFA should be considered as another safe and feasible adjuvant method for the palliative care of patients with advanced PHC. Since comparative studies are lacking, the choice of the local ablative method remains in each case an individual decision.

() 2021 S. Karger AG, Basel

\section{Introduction}

Cholangiocarcinomas (CCA) are the primary malignancies of the bile duct with an incidence that varies considerably, being 1-2/100,000/year in western countries but much higher in South-East Asia [1-4]. According to their localization, they are classified as either intrahepatic (originating from second or higher order bile ducts) or extrahepatic cholangiocarcinomas (eCCA). With the cystic duct confluence as reference point, eCCA are subdivided into distal CCA and perihilar cholangiocarcinoma (PHC). Since the Yale hepatologist Gerald Klatskin first characterized this type of CCA in 1965 [5], they are also called Klatskin tumors.

Patients with PHC are frequently diagnosed at an advanced stage with jaundice, weight loss, abdominal pain, or cholangitis, and due to locally advanced or metastatic 
stage, curative tumor resection is possible in only less than one-third of the patients $[4,6]$. Therefore, most patients are treated palliatively with gemcitabine and cisplatin as the current systemic first-line chemotherapy or with gemcitabine monotherapy in frail patients. Nevertheless, the median survival times are still less than 1 year, so that more targeted therapies are needed. Since molecular profiling of eCCA has recently identified key molecular drivers of tumorigenesis, biomarker-driven targeted therapies and immune checkpoint inhibitors are now available or currently under investigation for certain patients [7].

Since PHC cause obstructive cholestasis, it is an indispensable part of the palliative therapy concept to ensure adequate drainage of the biliary system in order to improve the patient's quality of life and to reduce the risk of obstructive cholangitis. This can be done using one or more plastic stents or self-expanding metal stents (SEMS), either covered or uncovered. In patients with PHC, placing a SEMS can be challenging, and especially covered SEMS might cause obstruction of segmental bile ducts. Therefore, multiple plastic stent placement is still often the method of choice in $\mathrm{PHC}$, notwithstanding that stent patency is generally longer for SEMS. Although plastic stents require regular replacement every $8-12$ weeks, they are particularly often preferred for biliary decompression, when a patient in a stable condition is scheduled for periodic intraductal ablative therapies [8].

\section{Intraductal Tumor Therapy for PHC}

In order to improve local tumor control and to secure biliary drainage by optimizing the stent patency, local ablative therapies like intraluminal biliary brachytherapy (ILBT) or photodynamic therapy (PDT) have been introduced since decades [8].

Early reports on ILBT were published in 1981, and therefore ILBT can be called the pioneer-technique used for intraductal ablative tumor therapy of CCA [9]. Nowadays, it is usually combined with either systemic chemotherapy and/or external-beam radiotherapy and performed as high-dose-rate brachytherapy in which 930$1600 \mathrm{cGy}$ are administered in one to four fractions over 1-2 days [10]. Following placement of one or two nasobiliary tubes, each with a metal-tipped, 4.7-Fr Varian intraluminal catheter with a dummy wire in the inner lumen, treatments are performed over 5-10 min after connecting the catheter with the VariSource remote afterloader containing a ${ }^{192} \mathrm{Ir}$ source of 5-10 curies. ILBT is a comparably safe technique, and there are promising smaller studies reporting improved survival rates for patients with CCA [11]. A recent multicenter study from Italy, however, showed that ILBT improved primarily the local tumor control, while the overall survival was not significantly better than with chemotherapy and external-beam radiotherapy alone [12]. Overall, ILBT has not yet become very widespread due to the additional equipment, logistics and time required and is only offered at a few specialized centers.

PDT employs phototoxic substances that are given intravenously and accumulate in the malignant tissue. Through a fiberoptic probe, which can be inserted into the affected bile duct endoscopically, laser light of a specific wavelength is emitted; this activates the photosensitizer and generates radical oxygen species leading to apoptosis and necrosis [8]. Ortner et al. [13] published in 2003 a prospective randomized study comparing stenting alone versus PDT and stenting in patients with irresectable CCA. They found that PDT was associated with longer survival (median 493 vs. 98 days, $p<0.0001$ ) and also improved biliary drainage and quality of life. A metaanalysis from 2012 pooled data from 170 patients from 6 prospective studies and reported that PDT and stenting was associated with a mean increase in the survival time of 265 days and an improvement of Karnofsky score compared with stenting alone [14]. Although several studies found evidence that PDT is more effective than stenting alone in the palliative treatment of eCCA, a possible antitumoral synergism of PDT and systemic chemotherapy has not been investigated until recently. But in a retrospective study from our group we found that PDT together with chemotherapy resulted in a significant longer overall survival than chemotherapy alone (20 months vs. 10 months, $p=0.022$ ) [15]. However, these promising efficacy data are somewhat constrained by the fact that phototoxicity of the photosensitizer is not acceptable for a number of patients, especially during summertime or in sunny regions of the world. In addition, a certain technical and logistical effort is required for PDT.

Taken together, there is evidence since the 1980s that the concept of intraductal ablative tumor therapy of irresectable extrahepatic CCA complementing a systemic antiproliferative approach is beneficial. However, due to side effects and the need for additional equipment, PDT and ILBT may not be the best practice. Notwithstanding that direct comparative studies are lacking, intraductal radiofrequency ablation (RFA) may be a technique that, with similar effectiveness, no longer has these disadvantages.

\section{Radiofrequency Ablation}

Radiofrequency is an electromagnetic wave frequency ranging from $10^{4}$ to $3 \times 10^{12} \mathrm{~Hz}$. Around 1891 , the French physician and biophysicist Jacques Arsene d'Arsonval and Serbian Croatian physicist Nikola Tesla indepen-
40

Visc Med 2021:37:39-47 DOI: 10.1159/000513970
Weismüller 


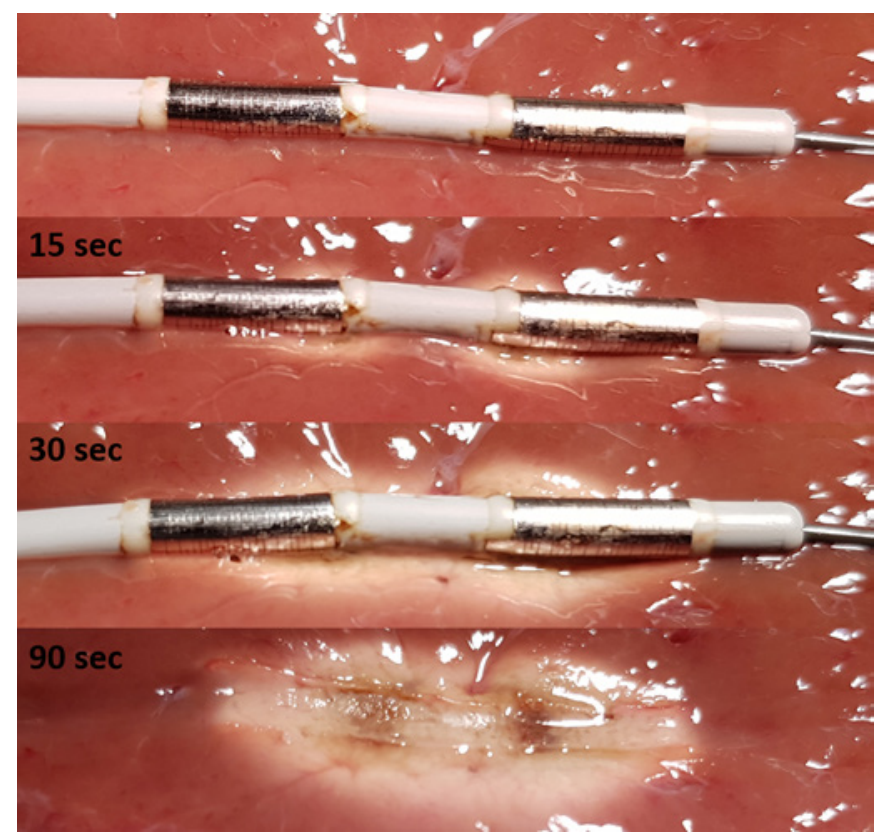

Fig. 1. Example images at consecutive time points from an ex vivo ablation procedure with the Habib ${ }^{\mathrm{TM}}$ EndoHPB catheter on porcine liver tissue. As HF generator we used the ERBE VIO $200 \mathrm{D}$ with the following setting: bipolar soft coagulation mode, effect 8 , $7 \mathrm{~W}$. The ablation zone starts forming at the electrodes and begins to fill the entire area between the electrodes after 20-30 s. Finally, the ablation zone has a consistent cylindrical shape.

dently of each other developed the idea, that high-frequency electromagnetic currents could have therapeutic effects. They found that alternating current frequencies above $20 \mathrm{kHz}$ are high enough to cause molecular frictional heating but without stimulating neuromuscular reaction (e.g., ventricular fibrillation) and electrolysis. At the beginning of the last century, the physicist William $\mathrm{T}$. Bovie produced in a collaboration with Harvey Cushing the first widely accepted RF generator for thermal coagulation [16]. This technique with a focused and brief RF energy deposition is since then widely used for the coagulation of surface lesions. In gastrointestinal endoscopy, it is a routine method for endoscopic resection and cutting techniques (e.g., submucosal dissection or mucosal resection, sphincterotomy or myotomy) or for superficial ablation like in Barrett's esophagus. While these techniques try to minimize collateral damage by avoiding energy dissipation into the surrounding tissues, RFA in contrast requires for the treatment of solid tumors a more volumetric and dispersed distribution of relatively mild RF energy to cause a greater extension of tissue destruction. During RFA, an electrical circuit between the body and a monopolar probe or between two bipolar probes is built, and ions within the tissue follow the alternating path of the current, creating frictional heat. A temperature above 48 $50{ }^{\circ} \mathrm{C}$ causes protein denaturation, cell dehydration, co- agulative necrosis, and cellular death. Since this effect is strongest in areas near the electrodes while tissues farther away are mainly heated by thermal conduction insufficient to cause necrosis, the volume of successfully ablated tissue is limited [17]. Improved RFA techniques with pulsed energy application, internal cooling systems, or optimization of the electrode design could help to partially overcome this limitation. The heat-sink phenomenon caused by a cooling effect from larger vessels near the target tissue can also lead to incomplete ablation.

To this day, the liver is the organ in which RFA has been used most intensively since the first pilot studies in the early 1990s [18]. According to current guidelines [19], RFA is considered standard of care for patients with small hepatocellular carcinoma not suitable for surgery. The therapeutic effect of RFA is probably not only caused by the thermal destruction of the tumor, but there is also increasing evidence that the cellular debris following RFA includes highly immunogenic intracellular components like heat shock proteins. It is assumed that this induces dendritic cell infiltration and tumor-specific T-cell responses and thereby triggers a systemic antitumor immune reaction [20-22].

\section{Intraductal RFA - Technical Considerations}

Following preliminary animal studies, which demonstrated the principal functionality of the method, the first phase 1 study on the use of intraductal RFA in patients with malignant biliary stricture (16 with pancreatic cancer, 6 with CCA) was published in 2011 by Steel et al. [23]. He used the Habib ${ }^{\mathrm{TM}}$ EndoHPB catheter (shown in Fig. 1), which can be introduced through standard working channels $(3.2 \mathrm{~mm})$ over a 0.035 -inch guidewire; $5 \mathrm{~mm}$ proximal to the leading edge, the probe has two ring electrodes $8 \mathrm{~mm}$ apart. This single-use bipolar RFA catheter can be connected with different widely used RFA generators and with an energy of $10 \mathrm{~W}$ for $90-120 \mathrm{~s}$ a circular coagulative necrosis with a length of $21-28 \mathrm{~mm}$ and a circumferential depth of $8-10 \mathrm{~mm}$ is induced in liver tissue [24]. Currently, the manufacturer (Boston Scientific Corp., Marlborough, MA, USA) recommends performing an ablation for $90 \mathrm{~s}$ with $10 \mathrm{~W}$ and to reduce the energy to $7 \mathrm{~W}$ for treatment above the biliary bifurcation or at the ampullary region. Following treatment, the probe should be left in place for $60 \mathrm{~s}$ to cool down. In the case of a malignant stenosis longer than $25 \mathrm{~mm}$, the ablation procedure can be repeated as often as needed to perform a complete ablation of the strictured area.

In 2017, Laleman et al. [25] reported on 18 patients (7 with pancreatic cancer, 11 with CCA) treated with a new intraductal RFA device, the ELRA ${ }^{\mathrm{TM}}$ RF catheter (STARmed and TaeWoong Medical, Korea), which al- 
Table 1. Comparison of the two available bipolar endoluminal RFA catheters

\begin{tabular}{lll}
\hline & Habib $^{\mathrm{TM}}$ EndoHPB & ELRA $^{\text {TM }}$ Electrode \\
\hline Manufacturer & Boston Scientific (USA) & STARmed (Korea) (distributed by TaeWoong) \\
\hline Diameter & $8 \mathrm{Fr}(2.6 \mathrm{~mm})$ & $7 \mathrm{Fr}(2.31 \mathrm{~mm})$ \\
\hline Size and design & $\begin{array}{l}24 \mathrm{~mm}-\text { with two bipolar electrodes }(8 \\
\text { mm each) }\end{array}$ & $\begin{array}{l}11 \text { or } 22 \mathrm{~mm} \text { - with two bipolar electrodes } \\
18 \text { or } 33 \mathrm{~mm} \text { - with four bipolar electrodes }\end{array}$ \\
\hline Radiofrequency generator & No specific generator required & Works only with VIVA Combo ${ }^{\text {TM }}($ STARmed) \\
\hline Energy control & Available & Available \\
\hline Temperature control & Not available & Available \\
\hline Alarm in case of poor electrode contact & No & Yes \\
\hline
\end{tabular}

lows temperature-controlled ablation. There are four different types of this 7-Fr bipolar RFA catheter available: 7 $\mathrm{mm}$ proximal to the leading edge, there are either two ring electrodes $4 \mathrm{~mm}$ in length and $3 \mathrm{~mm}$ apart or $8 \mathrm{~mm}$ in length and $6 \mathrm{~mm}$ apart; alternatively, the catheter is equipped with four ring electrodes each $3 \mathrm{~mm}$ in length and $2 \mathrm{~mm}$ apart or $6 \mathrm{~mm}$ in length and $3 \mathrm{~mm}$ apart, respectively. This design results in different presumptive electric coagulation lengths of $11,18,22$, or $33 \mathrm{~mm}$ and of ablation depths between 2 and $3 \mathrm{~mm}$ (circumferential depth of 6 and $8 \mathrm{~mm}$ ) following intraductal treatment in the bile-duct specimens of an in-vivo swine model [26]. The catheter can only be used with the VIVA combo ${ }^{\mathrm{TM}}$ RFA generator (Taewoong Medical, South Korea), which has versatile settings including power (range 0-200 W), temperature (range $5-95^{\circ} \mathrm{C}$ ), and time (range $10 \mathrm{~s}$ to 10 $\mathrm{min})$. Recommended settings for biliary RFA for this device are $7 \mathrm{~W}$ for the 11 - and $18-\mathrm{mm}$ catheter and $10 \mathrm{~W}$ for the 22- and 33- $\mathrm{mm}$ catheter for up to $2 \mathrm{~min}$. In the Temperature Mode, it is suggested to maintain a temperature of $75-85^{\circ} \mathrm{C}$. Furthermore, the VIVA combo ${ }^{\mathrm{TM}}$ RFA generator automatically stops the energy deposition when the electrodes lose contact to the lesion. This allows the treating physician to replace the catheter to a better position and avoids an inefficient procedure due to poor contact of the electrodes to the tissue.

According to the respective manufacturer's user instructions, the ELRA ${ }^{\mathrm{TM}}$ RF catheter is intended to be used for coagulation of tissue in malignant biliary obstruction, while the Habib ${ }^{\mathrm{TM}}$ EndoHPB catheter is intended to ablate both malignant and benign tissue in the pancreatic and biliary tracts. A summary of the main differences between the two available RFA catheters is shown in Table 1. It is presumed that the specific 4-ring design of the EL$\mathrm{RA}^{\mathrm{TM}} \mathrm{RF}$ catheter allows a more targeted application of the thermal energy and that due to the capacity to control and maintain the locally applied temperature via a dedicated and feedback-sensing generator, the risk of supra- therapeutic tissue damage is lower [25]. This could result in a reduced incidence of complications like pancreatitis (especially when RFA is performed in the ampullary region) or of hemobilia, vascular thrombosis, or perforation. However, so far there are no comparative studies published that present evidence for a significant advantage of one of the two systems regarding efficacy or safety.

\section{Indications of Intraductal RFA}

Intraductal RFA can be indicated not only for the treatment of malignant strictures (including CCA or pancreatic carcinoma but also metastasis from other tumors); it is also used to treat occluded SEMS, to treat remnant tissue due to bile duct ingrowth of papillary adenoma following papillectomy and in rare cases to treat benign strictures: A retrospective case-control study evaluated the role of additional RFA for the ablation of malignant tissue-ingrowth in SEMS and reported an improved stent patency rate with RFA compared to plastic stenting alone (119.5 vs. 65.3 days, $p=0.03$ ) [27]. However, data from in vivo and in vitro models [28] show that the ablation depth after intraductal RFA is markedly reduced in the bile duct section, where a SEMS was placed, so that an effect on the malignant tumor itself is unlikely. A small pilot study on 9 patients with benign biliary strictures of different etiologies [29] suggested that intraductal RFA was a safe and effective treatment alternative, especially for refractory cases. Two multicenter studies, one with a retrospective design and 14 patients [30] and the other with a prospective design and 20 patients [31] analyzed efficacy and safety of intraductal RFA for the treatment of ampullary neoplasms with intraductal extension. They reported complete eradication of neoplastic tissue in 92 and 70\%, but also adverse events in about $40 \%$ of the patients. Especially due to the risk of postinterventional pancreatitis or biliary strictures, it is recommended to place plastic 
Fig. 2. Cholangiographic images of a patient with irresectable PHC (Bismuth IV, histologically proven; A) treated for 5 years with systemic chemotherapy (gemcitabine \& cisplatin) and repeated intraductal RFA (B) every 3 months followed by plastic stenting. The malignant hilar stricture was kept stable and open for 4 years (C). In the 5 th year, we noticed a progression of the stricture (D), and 4 weeks after the last RFA procedure, arterial intrabiliary bleeding from a pseudoaneurysm of the right hepatic artery occurred (E). Although this was controlled through embolization and biliary stents (F), a further RFA was not possible anymore, and the patient finally died in April 2018 due to liver failure.
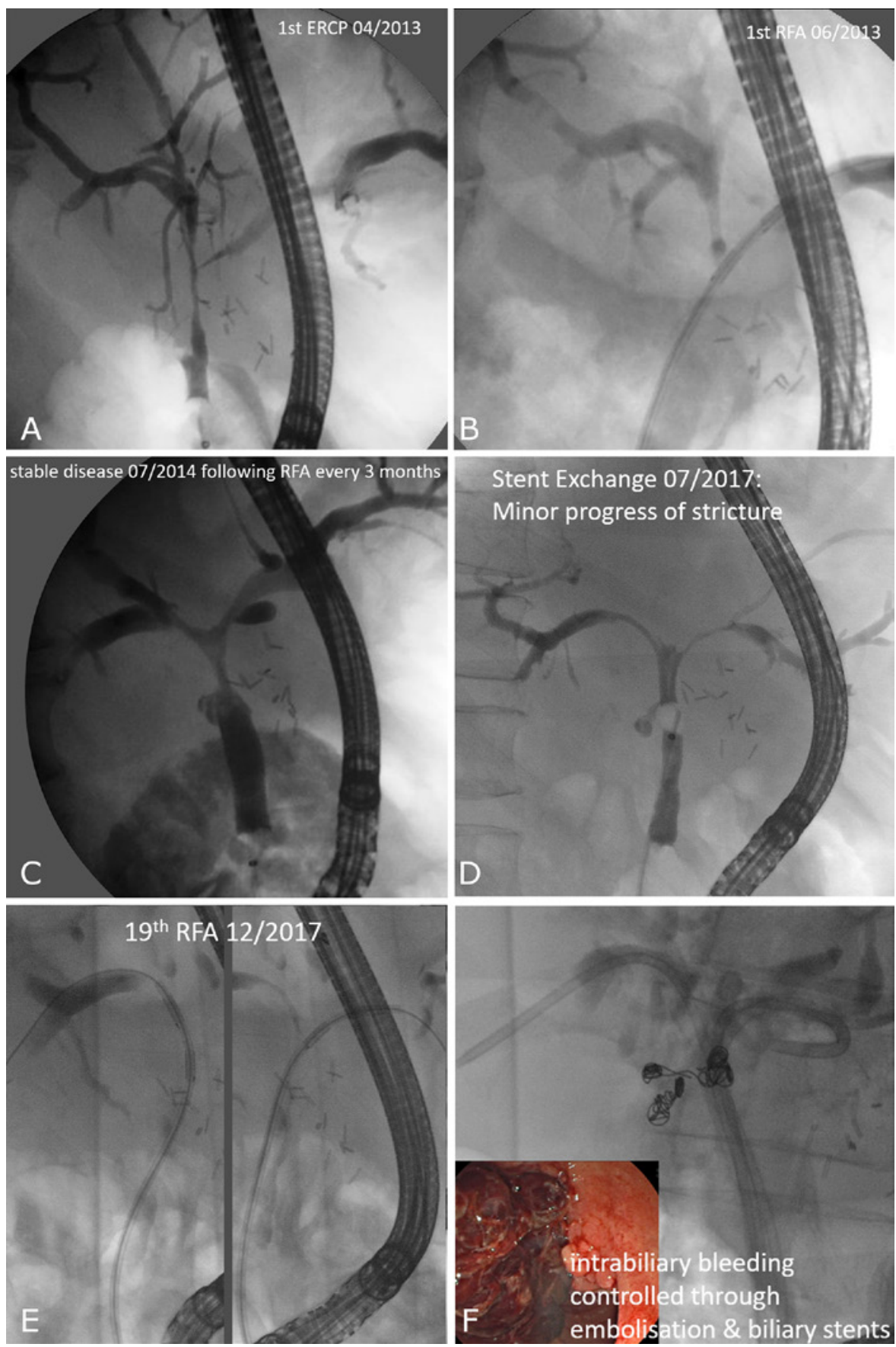

stents into the pancreatic duct and the common bile duct following RFA in the papillary region. Furthermore, a shorter duration of energy application (30 s) and a lower energy output $(7 \mathrm{~W})$ should be considered.

\section{Efficacy and Safety of Intraductal RFA for PHC}

Inoperable malignant bile duct strictures are the main indication for intraductal RFA. A typical case from our Department is shown in Figure 2. After the first report from Steel et al. [23] in 2011 more than 40 mainly retro- spective studies have been published, which evaluated the use of RFA in malignant biliary obstruction with conflicting results. These studies markedly differ in design (retrospective or prospective observational studies, randomized controlled trials (RCT)), concomitant therapy (plastic or metal stents, chemotherapy), and included etiologies (pancreatic head cancers, eCCA, gallbladder cancer, liver metastasis, malignant hilar lymphadenopathy). But also regarding the analyzed endpoints (stent patency rate, overall survival or progression free survival, complication rate), the studies are not comparable. Furthermore, the number and interval of RFA sessions varies substantially 


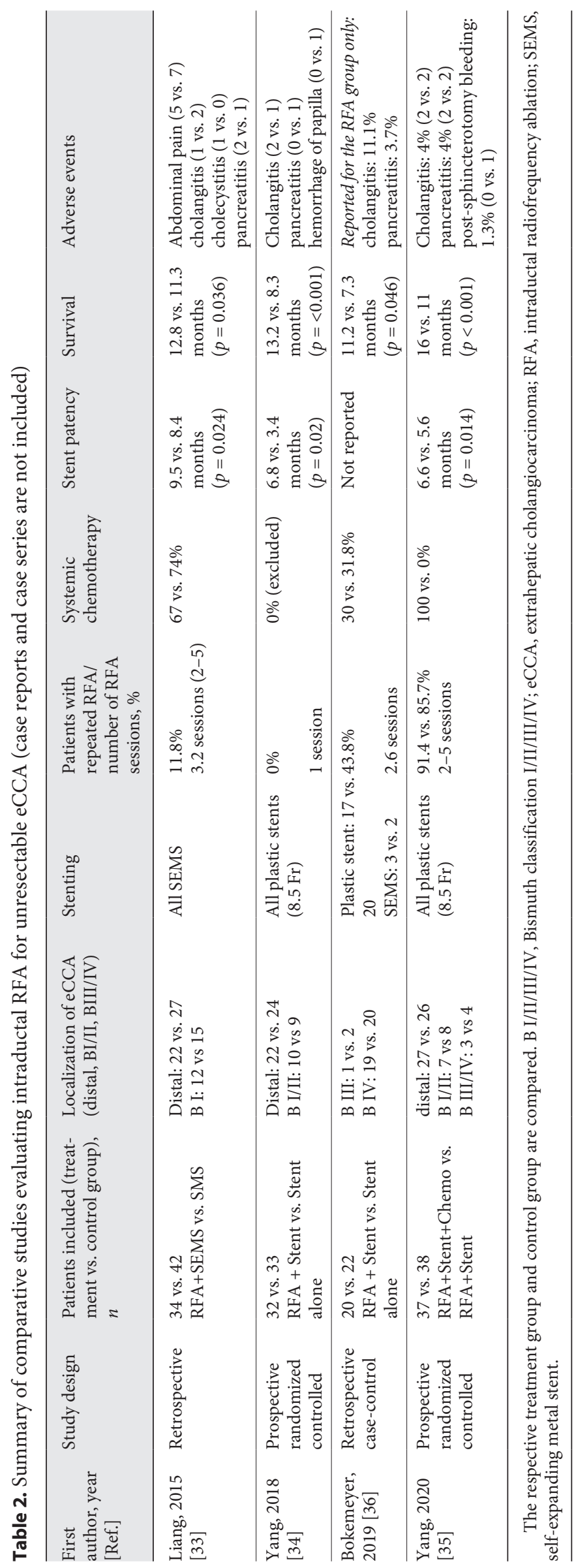

between the studies. In order to analyze the efficacy and safety of biliary SEMS placement with or without previous intraductal RFA in patients with malignant biliary strictures, a systematic meta-analysis from 2018 included 9 studies with altogether 239 versus 266 patients [32]. RFA was performed only once in $78 \%$ of the included studies, and adjuvant chemotherapy was given less frequently in the RFA group ( 17.5 vs. $21 \%, p=0.03$ ). Nevertheless, the pooled mean stent patency was 50.6 days longer in the treatment group $(p=0.002)$ and the pooled median survival rate was better in the RFA group (285 vs. 248 days; $p<0.001)$. AE rates did not differ significantly for cholangitis (6.2 vs. $5.2 \%$ ), pancreatitis (2.1 vs. $1.5 \%$ ), and hemobilia (3.8 vs. $1.9 \%$ ), but postinterventional abdominal pain was reported more frequently in the RFA group (31 vs. $20 \%, p=0.003$ ). However, it is unclear whether these findings would also apply to PHCs primarily treated with ERC, since RFA was performed percutaneously in about $50 \%$ of included patients and since patients with pancreatic carcinoma were included as well.

Until today, only 4 studies have been published, which analyzed the influence of intraductal RFA on survival specifically for unresectable eCCA (Table 2). A case-control study from 2015 [33] compared retrospectively the survival of patients with unresectable Bismuth type I PHC $(n=27[35 \%])$ and with distal eCCA $(n=49$ [65\%]), who were treated with SEMS-implantation with $(n=34)$ or without $(n=42)$ a previous RFA. The groups were comparable with regard to tumor stage, demographic data, and adjuvant chemotherapy ( 67 vs. $74 \%$, ns), but the median stent patency was significantly longer for the RFA+SEMS group (9.5 vs. 8.4 months; $p=0.024$ ) and the overall survival was significantly improved $(p=0.036)$. Yang et al. [34] published in 2018 the first prospective RCT evaluating a survival benefit of RFA for patients with inoperable eCCA. 65 patients with unresectable Bismuth type I and II PHC $(n=19)$ or with a distal eCCA $(n=46)$ were randomized to receive either RFA followed by plastic stent insertion (8.5 Fr) $(n=32)$ or plastic stent alone $(n=33)$. Patients in the RFA group showed a significantly longer stent patency (6.8 vs. 3.4 months, $p=0.02$ ) and a significantly longer overall survival after randomization than control patients (13.2 vs. 8.3 months; $p<0.001$ ), while the rate of adverse events was comparable (6.3 vs. $9.1 \%)$. It is nonetheless questionable whether these promising results are transferable into a real-world setting since Bismuth type III and IV PHC, severe liver dysfunction, and systemic chemotherapy were exclusion criteria. Interestingly, the same group recently published a prospective RCT [35] that evaluated the clinical efficacy and safety of intraductal RFA in combination $(n=37)$ with a novel oral 5-fluorouracil compound (S-1) versus intraductal RFA alone $(n=38)$ for the treatment of unresectable locally advanced eCCA ( $70 \%$ distal CCA). While the 


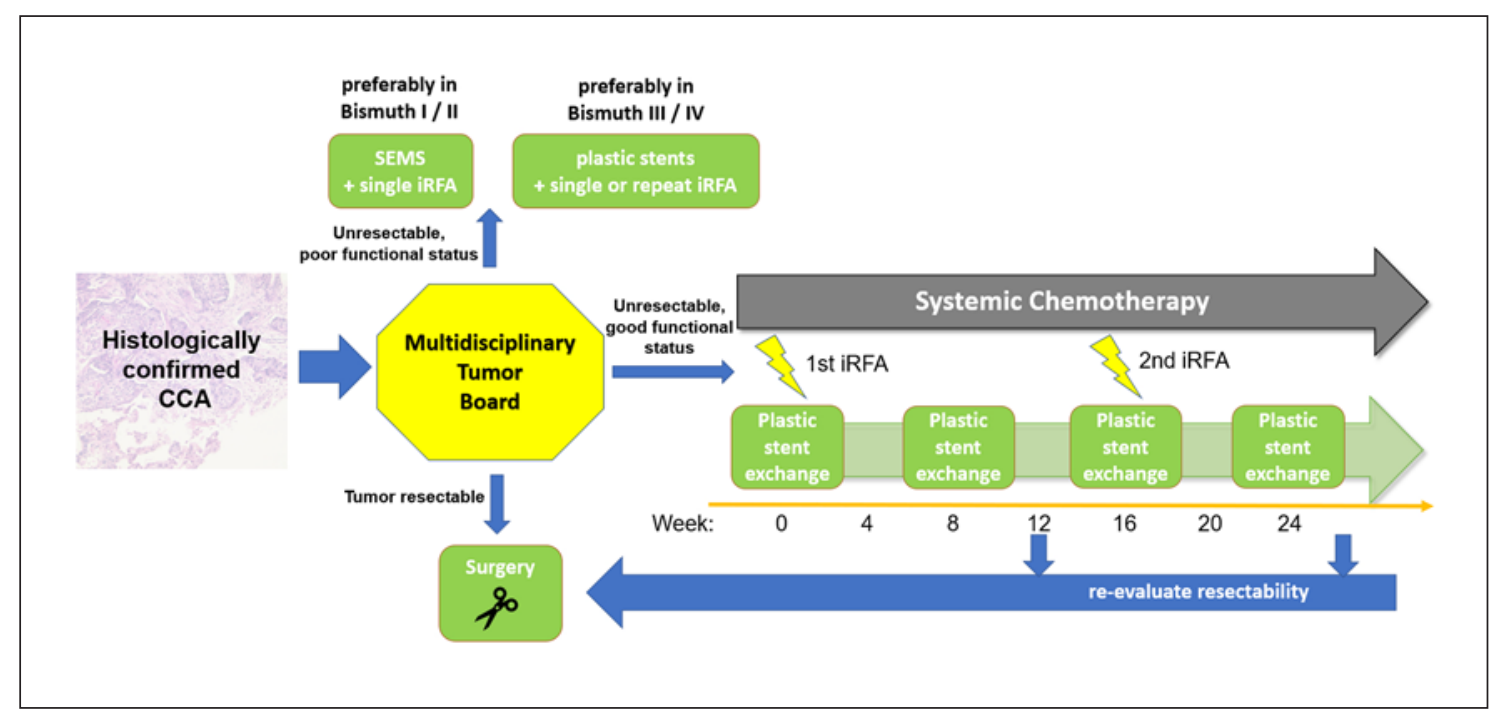

Fig. 3. Proposal of an algorithm on the possible role of intraductal RFA as a treatment approach for extrahepatic CCA.

incidence of ERCP-related adverse events did not differ significantly, the median overall survival (16 vs. 11 months, $p<0.001)$ as well as the stent patency time (6.6 vs. 5.6 months, $p=0.014$ ) was significantly longer in the combination group. As mentioned above, experimental data show that the cellular debris following RFA can trigger a systemic antitumor immune reaction [20-22]; hence, it is presumed that there are synergistic effects of chemotherapy and intraductal RFA, which lead to the observed longer survival in the combination group compared to RFA or chemotherapy alone. Against the background that previous studies predominantly included distal or Bismuth type I or II eCCA, the retrospective case-control study by Bokemeyer et al. [36] is of particular interest. They compared retrospectively 20 patients with Bismuth type III and IV PHC receiving intraductal RFA + stenting (85\% plastic and 15\% metal stent) with a matched control group $(n=22)$ treated with stent placement only (91\% plastic and 9\% metal stent), and the combination group showed a significantly longer survival (342 vs. 221 days, $p=0.046$ ). Most frequent complications following RFA were cholangitis (11.1\%) and pancreatitis (3.7\%).

\section{Conclusions and Unanswered Questions}

Intraductal RFA is another promising tool in the therapeutic armamentarium for the endoscopic management and tumor ablation in patients with PHC. Figure 3 shows a proposed algorithm on intraductal RFA as a treatment approach for extrahepatic CCA. First study results suggest that RFA is at least as effective as comparable meth- ods like PDT or ILBT but has fewer systemic side effects and requires less logistical or infrastructural effort. However, while RFA requires direct contact of the electrodes with the tissue for ablation, laser light has the advantage to refract through bile and thus treat also malignant tissue that is not in direct proximity to the laser fiber. Incomplete contact of the electrode surface to an irregular malignant stenosis could lead to an incomplete ablation, but only the ELRA ${ }^{\mathrm{TM}}$ RF catheter has the capability to give feedback on incomplete electrode contact. Compared to the very thin PDT laser fiber, the RFA catheter has a significantly larger diameter and is much stiffer, which makes it sometimes impossible to place it within a very narrow or very angled malignant stricture. Nevertheless, while comparative studies on the safety and efficacy of RFA vs. PDT are lacking, a retrospective analysis found that the short-term therapeutic effects and the safety profile were comparable between intraductal RFA and PDT for treatment of PHC [37]; but to answer the question which treatment modality (RFA, PDT, or ILBT) is optimal in which situation, multicenter prospective RCT are needed. It is also unclear if the theoretical advantages of temperature-controlled intraductal RFA are associated with improved outcome, since studies comparing both available RFA catheters are lacking. A possible role of intraductal RFA in a neoadjuvant strategy before liver transplantation or liver resection remains as well to be elucidated. Therefore, RFA should be considered as part of the therapeutic concept in future study protocols in which neoadjuvant strategies for PHC are evaluated. The safety profile of intraductal RFA appears acceptable in the context of a malignant disease, but in order to become a routine component of the adjuvant therapy for PHC, 
multicenter prospective RCT are needed to develop standardized protocols for treatment with regard to energy settings, number, and frequency of treatment sessions.

\section{Conflict of Interest Statement}

Tobias J. Weismüller has received speaker fees from Boston Scientific, Cook-Medical, and Fujifilm.

\section{Funding Sources}

None.

\section{Author Contributions}

Tobias J. Weismüller has written the manuscript.

\section{References}

1 Blechacz B, Komuta M, Roskams T, Gores GJ. Clinical diagnosis and staging of cholangiocarcinoma. Nat Rev Gastroenterol Hepatol. 2011 Aug;8(9):512-22.

2 von Hahn T, Ciesek S, Wegener G, Plentz RR, Weismüller TJ, Wedemeyer $\mathrm{H}$, et al. Epidemiological trends in incidence and mortality of hepatobiliary cancers in Germany. Scand J Gastroenterol. 2011 Sep;46(9):1092-8.

3 Blechacz B. Cholangiocarcinoma: Current Knowledge and New Developments. Gut Liver. 2017 Jan;11(1):13-26.

4 Schweitzer N, Fischer M, Kirstein MM, Berhane S, Kottas M, Sinn M, et al. Risk estimation for biliary tract cancer: development and validation of a prognostic score. Liver Int. 2017 Dec;37(12):1852-60.

5 Klatskin G. Adenocarcinoma of the Hepatic Duct at Its Bifurcation within the Porta Hepatis. An Unusual Tumor with Distinctive Clinical and Pathological Features. Am J Med. 1965 Feb;38(2):241-56.

6 Hartog H, Ijzermans JN, van Gulik TM, Groot Koerkamp B. Resection of Perihilar Cholangiocarcinoma. Surg Clin North Am. 2016 Apr;96(2):247-67.

7 Fostea RM, Fontana E, Torga G, Arkenau HT. Recent Progress in the Systemic Treatment of Advanced/Metastatic Cholangiocarcinoma. Cancers (Basel). 2020 Sep;12(9):E2599.

8 Wang AY, Yachimski PS. Endoscopic Management of Pancreatobiliary Neoplasms. Gastroenterology. 2018 May;154(7):1947-63.

9 Benjamin IS, McPherson GA, Blumgart LH. Iridium-192 wire for hilar cholangiocarcinoma. Lancet. 1981 Sep;2(8246):582-3.

10 Mukewar S, Gupta A, Baron TH, Gores G, Furutani $\mathrm{K}$, Haddock MG, et al. Endoscopically inserted nasobiliary catheters for high doserate brachytherapy as part of neoadjuvant therapy for perihilar cholangiocarcinoma. Endoscopy. 2015 Oct;47(10):878-83.

11 Karani J, Fletcher M, Brinkley D, Dawson JL, Williams R, Nunnerley $H$. Internal biliary drainage and local radiotherapy with iridium-192 wire in treatment of hilar cholangiocarcinoma. Clin Radiol. 1985 Nov;36(6):6036.

12 Autorino R, Bisiello S, Pappalardi B, Privitera $\mathrm{V}$, Buwenge $\mathrm{M}$, Piccolo $\mathrm{F}$, et al. Intraluminal Brachytherapy in Unresectable Extrahepatic Biliary Duct Cancer: An Italian Pooled Analysis. Anticancer Res. 2020 Jun;40(6):3417-21.
13 Ortner ME, Caca K, Berr F, Liebetruth J, Mansmann U, Huster D, et al. Successful photodynamic therapy for nonresectable cholangiocarcinoma: a randomized prospective study. Gastroenterology. 2003 Nov;125(5):1355-63.

14 Leggett CL, Gorospe EC, Murad MH, Montori VM, Baron TH, Wang KK. Photodynamic therapy for unresectable cholangiocarcinoma: a comparative effectiveness systematic review and meta-analyses. Photodiagn Photodyn Ther. 2012 Sep;9(3):189-95.

15 Gonzalez-Carmona MA, Bolch M, Jansen C, Vogt A, Sampels M, Mohr RU, et al. Combined photodynamic therapy with systemic chemotherapy for unresectable cholangiocarcinoma. Aliment Pharmacol Ther. 2019 Feb; 49(4):437-47.

16 Ni Y, Mulier S, Miao Y, Michel L, Marchal G. A review of the general aspects of radiofrequency ablation. Abdom Imaging. 2005 JulAug;30(4):381-400.

17 Alvarez-Sánchez MV, Napoléon B. Review of endoscopic radiofrequency in biliopancreatic tumours with emphasis on clinical benefits, controversies and safety. World J Gastroenterol. 2016 Oct;22(37):8257-70.

18 McGahan JP, Browning PD, Brock JM, Tesluk $\mathrm{H}$. Hepatic ablation using radiofrequency electrocautery. Invest Radiol. 1990 Mar;25(3): 267-70.

19 Galle PR, Forner A, Llovet JM, Mazzaferro V, Piscaglia F, Raoul JL, et al.; European Association for the Study of the Liver. Electronic address: easloffice@easloffice.eu; European Association for the Study of the Liver. Electronic address eee, European Association for the Study of the L. EASL Clinical Practice Guidelines: management of hepatocellular carcinoma. J Hepatol. 2018 Jul;69(1):182236.

20 Ali MY, Grimm CF, Ritter M, Mohr L, Allgaier HP, Weth R, et al. Activation of dendritic cells by local ablation of hepatocellular carcinoma. J Hepatol. 2005 Nov;43(5):81722.

21 Teng LS, Jin KT, Han N, Cao J. Radiofrequency ablation, heat shock protein 70 and potential anti-tumor immunity in hepatic and pancreatic cancers: a minireview. Hepatobiliary Pancreat Dis Int. 2010 Aug;9(4):361-5.

22 Haen SP, Pereira PL, Salih HR, Rammensee HG, Gouttefangeas C. More than just tumor destruction: immunomodulation by thermal ablation of cancer. Clin Dev Immunol. 2011; 2011:160250.
23 Steel AW, Postgate AJ, Khorsandi S, Nicholls J, Jiao L, Vlavianos P, et al. Endoscopically applied radiofrequency ablation appears to be safe in the treatment of malignant biliary obstruction. Gastrointest Endosc. 2011 Jan; 73(1):149-53.

24 Itoi $\mathrm{T}$, Isayama $\mathrm{H}$, Sofuni A, Itokawa F, Tamura $\mathrm{M}$, Watanabe $\mathrm{Y}$, et al. Evaluation of effects of a novel endoscopically applied radiofrequency ablation biliary catheter using an exvivo pig liver. J Hepatobiliary Pancreat Sci. 2012 Sep;19(5):543-7.

25 Laleman W, van der Merwe S, Verbeke L, Vanbeckevoort D, Aerts R, Prenen H, et al. A new intraductal radiofrequency ablation device for inoperable biliopancreatic tumors complicated by obstructive jaundice: the IGNITE-1 study. Endoscopy. 2017 Oct;49(10): 977-82.

26 Cho JH, Lee KH, Kim JM, Kim YS, Lee DH, Jeong S. Safety and effectiveness of endobiliary radiofrequency ablation according to the different power and target temperature in a swine model. J Gastroenterol Hepatol. 2017 Feb;32(2):521-6.

27 Kadayifci A, Atar M, Forcione DG, Casey BW, Kelsey PB, Brugge WR. Radiofrequency ablation for the management of occluded biliary metal stents. Endoscopy. 2016 Dec; 48(12):1096-101.

28 Yoon WJ, Kim YT, Daglilar ES, Mino-Kenudson M, Brugge WR. Evaluation of bipolar radiofrequency ablation for occluded self-expandable metal stents in the bile duct: in vivo and in vitro study. Endoscopy. 2015 Dec; 47(12):1167-70.

$29 \mathrm{Hu}$ B, Gao DJ, Wu J, Wang TT, Yang XM, Ye $\mathrm{X}$. Intraductal radiofrequency ablation for refractory benign biliary stricture: pilot feasibility study. Dig Endosc. 2014 Jul;26(4):581-5.

30 Rustagi T, Irani S, Reddy DN, Abu Dayyeh BK, Baron TH, Gostout CJ, et al. Radiofrequency ablation for intraductal extension of ampullary neoplasms. Gastrointest Endosc. 2017 Jul;86(1):170-6.

31 Camus M, Napoléon B, Vienne A, Le Rhun M, Leblanc S, Barret M, et al. Efficacy and safety of endobiliary radiofrequency ablation for the eradication of residual neoplasia after endoscopic papillectomy: a multicenter prospective study. Gastrointest Endosc. 2018 Sep; 88(3):511-8. 
32 Sofi AA, Khan MA, Das A, Sachdev M, Khuder S, Nawras A, et al. Radiofrequency ablation combined with biliary stent placement versus stent placement alone for malignant biliary strictures: a systematic review and meta-analysis. Gastrointest Endosc. 2018 Apr;87(4): 944-951.e1.

33 Liang H, Peng Z, Cao L, Qian S, Shao Z. Metal Stenting with or without Endobiliary Radiofrequency Ablation for Unresectable Extrahepatic Cholangiocarcinoma. J Cancer Ther. 2015;06(11):981-92.
34 Yang J, Wang J, Zhou H, Zhou Y, Wang Y, Jin $\mathrm{H}$, et al. Efficacy and safety of endoscopic radiofrequency ablation for unresectable extrahepatic cholangiocarcinoma: a randomized trial. Endoscopy. 2018 Aug;50(8):751-60.

35 Yang J, Wang J, Zhou H, Wang Y, Huang H, Jin $\mathrm{H}$, et al. Endoscopic radiofrequency ablation plus a novel oral 5-fluorouracil compound versus radiofrequency ablation alone for unresectable extrahepatic cholangiocarcinoma. Gastrointest Endosc. 2020 Dec;92(6):1204-1212.e1.
36 Bokemeyer A, Matern P, Bettenworth D, Cordes F, Nowacki TM, Heinzow H, et al. Endoscopic Radiofrequency Ablation Prolongs Survival of Patients with Unresectable Hilar Cholangiocellular Carcinoma - A Case-Control Study. Sci Rep. 2019 Sep;9(1):13685.

37 Schmidt A, Bloechinger M, Weber A, Siveke J, von Delius S, Prinz C, et al. Short-term effects and adverse events of endoscopically applied radiofrequency ablation appear to be comparable with photodynamic therapy in hilar cholangiocarcinoma. United European Gastroenterol J. 2016 Aug;4(4):570-9. 